J. Korean Math. Soc. 49 (2012), No. 1, pp. 139-151

http://dx.doi.org/10.4134/JKMS.2012.49.1.139

\title{
CROSS COMMUTATORS ON BACKWARD SHIFT INVARIANT SUBSPACES OVER THE BIDISK II
}

\author{
Kei Ji Izuchi And Kou Hei IzuchI
}

\begin{abstract}
In the previous paper, we gave a characterization of backward shift invariant subspaces of the Hardy space over the bidisk on which $\left[S_{z^{n}}, S_{w}^{*}\right]=0$ for a positive integer $n \geq 2$. In this case, it holds that $S_{z^{n}}=c I$ for some $c \in \mathbb{C}$. In this paper, it is proved that if $\left[S_{\varphi}, S_{w}^{*}\right]=0$ and $\varphi \in H^{\infty}\left(\Gamma_{z}\right)$, then $S_{\varphi}=c I$ for some $c \in \mathbb{C}$.
\end{abstract}

\section{Introduction}

Let $\Gamma^{2}$ be the 2-dimensional unit torus. We write $(z, w)=\left(e^{i s}, e^{i t}\right)$ for variables in $\Gamma^{2}=\Gamma_{z} \times \Gamma_{w}$. Let $L^{2}=L^{2}\left(\Gamma^{2}\right)$ be the usual Lebesgue space on $\Gamma^{2}$ with the norm

$$
\|f\|_{2}=\left(\int_{0}^{2 \pi} \int_{0}^{2 \pi}\left|f\left(e^{i s}, e^{i t}\right)\right|^{2} \frac{d s d t}{(2 \pi)^{2}}\right)^{1 / 2} .
$$

With the usual inner product, $L^{2}$ is a Hilbert space. Let $H^{2}=H^{2}\left(\Gamma^{2}\right)$ be the Hardy space over $\Gamma^{2}$. We denote by $H^{2}\left(\Gamma_{z}\right)$ and $H^{2}\left(\Gamma_{w}\right)$ the Hardy spaces on the unit circle $\Gamma$ in variables $z$ and $w$, respectively. We think of $H^{2}\left(\Gamma_{z}\right)$ and $H^{2}\left(\Gamma_{w}\right)$ as closed subspaces $H^{2}$. For each $f \in H^{2}$, we can write $f$ as

$$
f=\sum_{i=0}^{\infty} \oplus f_{i}(w) z^{i}, \quad f_{i}(w) \in H^{2}\left(\Gamma_{w}\right) .
$$

Let $P$ be the orthogonal projection from $L^{2}$ onto $H^{2}$. For a closed subspace $M$ of $L^{2}$, we denote by $P_{M}$ the orthogonal projection from $L^{2}$ onto $M$. For a function $\psi \in L^{\infty}$, the Toeplitz operator $T_{\psi}$ on $H^{2}$ is defined by $T_{\psi} f=P(\psi f)$ for $f \in H^{2}$. It is well known that $T_{\psi}^{*}=T_{\bar{\psi}}$, and $T_{\varphi(z)}^{*} T_{\psi(w)}=T_{\psi(w)} T_{\varphi(z)}^{*}$ for every $\varphi(z) \in H^{\infty}\left(\Gamma_{z}\right)$ and $\psi(w) \in H^{\infty}\left(\Gamma_{w}\right)$. A function $f \in H^{2}$ is called inner if $|f|=1$ on $\Gamma^{2}$ almost everywhere. A nonzero closed subspace $M$ of $H^{2}$ is

Received September 20, 2010; Revised December 17, 2010.

2010 Mathematics Subject Classification. Primary 47A15, 32A35; Secondary 47 B35.

Key words and phrases. backward shift invariant subspace, invariant subspace, Hardy space, cross commutator.

The first author is partially supported by Grant-in-Aid for Scientific Research (No.16340037), Japan Society for the Promotion of Science. 
called invariant if $z M \subset M$ and $w M \subset M$. In one variable case, the well known Beurling theorem [2] says that an invariant subspace $M$ of $H^{2}\left(\Gamma_{z}\right)$ has a form $M=q(z) H^{2}\left(\Gamma_{z}\right)$, where $q(z)$ is an inner function. In two variable case, the structure of invariant subspaces of $H^{2}$ is extremely complicated, see $[3,10]$.

Let $M$ be an invariant subspace of $H^{2}$ with $M \neq\{0\}$ and $M \neq H^{2}$. Then $T_{z}^{*}\left(H^{2} \ominus M\right) \subset H^{2} \ominus M$ and $T_{w}^{*}\left(H^{2} \ominus M\right) \subset H^{2} \ominus M$. In this paper, we write

$$
N=H^{2} \ominus M \text {. }
$$

Usually, $N$ is called a backward shift invariant subspace of $H^{2}$. See $[1,9]$ for studies of backward shift invariant subspaces over the unit circle $\Gamma$.

For a function $\psi \in L^{\infty}$, we denote by $R_{\psi}$ the operator on $M$ defined by $R_{\psi} f=P_{M}(\psi f)$ for $f \in M$. It holds $R_{\psi}^{*}=R_{\bar{\psi}}$ and $R_{z}=\left.T_{z}\right|_{M}$. We denote by $\left[R_{z}, R_{w}^{*}\right]$ the cross commutator of $R_{z}$ and $R_{w}$, that is, $\left[R_{z}, R_{w}^{*}\right]=R_{z} R_{w}^{*}-$ $R_{w}^{*} R_{z}$. In [8], Mandrekar proved that $\left[R_{z}, R_{w}^{*}\right]=0$ if and only if $M$ is Beurling type, that is, $M=q H^{2}$ for some inner function $q$ on $\Gamma^{2}$. This is a nice characterization of Beurling type invariant subspaces of $H^{2}$. More generally, in [4] the authors proved that $\left[R_{z}, R_{w}^{*}\right]=0$ if and only if $\left[R_{\psi_{1}(z)}, R_{\psi_{2}(w)}^{*}\right]=0$ for nonconstant functions $\psi_{1}(z), \psi_{2}(w) \in H^{\infty}(\Gamma)$.

We define the operator $S_{\psi}$ on $N$ by $S_{\psi} f=P_{N}(\psi f)$ for $f \in N$. Then we have $S_{\psi}^{*}=S_{\bar{\psi}}$ and $S_{z}^{*}=\left.T_{z}^{*}\right|_{N}$. In [6], it is proved that $\left[S_{z}, S_{w}^{*}\right]=0$ if and only if $N$ has one of the following forms;

$$
\begin{aligned}
& \cdot N=H^{2} \ominus q_{1}(z) H^{2} \\
& \text { - } N=H^{2} \ominus q_{2}(w) H^{2} \\
& \text { - } N=\left(H^{2} \ominus q_{1}(z) H^{2}\right) \cap\left(H^{2} \ominus q_{2}(w) H^{2}\right)
\end{aligned}
$$

for nonconstant one variable inner functions $q_{1}(z)$ and $q_{2}(w)$. In [7], it is shown that the condition $\left[S_{z^{2}}, S_{w}^{*}\right]=0$ does not imply $\left[S_{z}, S_{w}^{*}\right]=0$. In [5], the authors proved that for $n \geq 2,\left[S_{z^{n}}, S_{w}^{*}\right]=0$ if and only if one of the following conditions holds;

(i) $\left[S_{z}, S_{w}^{*}\right]=0$,

(ii) $S_{z^{n}} S_{w}^{*}=0$,

(iii) there exists a Blaschke product $b(z)$ with

$$
b(z)=\prod_{j=1}^{n} \frac{z-\alpha_{j}}{1-\bar{\alpha}_{j} z}, \quad 0<\left|\alpha_{j}\right|<1,
$$

where $\alpha_{i} \neq \alpha_{j}$ for every $i, j$ with $i \neq j$ and $\alpha_{1}^{n}=\alpha_{2}^{n}=\cdots=\alpha_{n}^{n}$ such that $N \subset H^{2} \ominus b(z) H^{2}$.

In [7, Theorem 2.2], it is proved that (ii) holds if and only if either $N \subset H^{2}\left(\Gamma_{z}\right)$ or $N \subset H^{2} \ominus z^{n} H^{2}$. If $N \subset H^{2}\left(\Gamma_{z}\right)$, then we have $\left[S_{z}, S_{w}^{*}\right]=0$. Moreover, in [5] it is proved that if $\left[S_{z^{n}}, S_{w}^{*}\right]=0$ and $\left[S_{z}, S_{w}^{*}\right] \neq 0$, then $M \cap H^{\infty}\left(\Gamma_{z}\right)=$ $\theta(z) H^{\infty}\left(\Gamma_{z}\right)$ for an inner function $\theta(z)$, and $z^{n} \in \mathbb{C}+\theta(z) H^{\infty}\left(\Gamma_{z}\right)$. In this case, we have $S_{z^{n}}=c I$ for some $c \in \mathbb{C}$.

The purpose of this paper is to generalize the above phenomeron. Let $\varphi(z) \in H^{\infty}\left(\Gamma_{z}\right)$ be a nonconstant function. Suppose that $\left[S_{\varphi(z)}, S_{w}^{*}\right]=0$ and 
$\left[S_{z}, S_{w}^{*}\right] \neq 0$. In Section 2, we prove that $M \cap H^{\infty}\left(\Gamma_{z}\right) \neq\{0\}$ and $M \cap H^{2}\left(\Gamma_{z}\right) \neq$ $H^{2}\left(\Gamma_{z}\right)$. Hence by the Beurling theorem, $M \cap H^{2}\left(\Gamma_{z}\right)=\theta(z) H^{2}\left(\Gamma_{z}\right)$ for a nonconstant inner function $\theta(z)$. Thus we get $\theta(z) H^{2} \subset M$. Write

$$
M_{\theta}=M \ominus \theta(z) H^{2} .
$$

We prove that $M_{\theta} \neq\{0\}$ and $T_{\varphi(z)}^{*} M_{\theta} \subset M_{\theta}$. In another word, $\varphi(z) N \subset$ $N \oplus \theta(z) H^{2}$ holds. In Section 3, we study on the one variable Hardy space $H^{2}\left(\Gamma_{z}\right)$. Let $N_{1}, N_{2}$ be backward shift invariant subspaces of $H^{2}\left(\Gamma_{z}\right)$ satisfying $\{0\} \neq N_{2} \varsubsetneqq N_{1} \neq H^{2}\left(\Gamma_{z}\right)$. It is proved that $\varphi(z) N_{2} \subset N_{2} \oplus\left(H^{2}\left(\Gamma_{z}\right) \ominus N_{1}\right)$ if and only if $\varphi(z) \in \mathbb{C}+\left(H^{2}\left(\Gamma_{z}\right) \ominus N_{1}\right)$. As applications of these facts, in Section 4 we prove that $\varphi(z) \in \mathbb{C}+\theta(z) H^{\infty}\left(\Gamma_{z}\right)$ and $S_{\varphi}=c I$ for some $c \in \mathbb{C}$.

\section{Equivalent conditions for $\left[S_{\varphi(z)}, S_{w}^{*}\right]=0$}

Let $N$ be a backward shift invariant subspace of $H^{2}$ with $N \neq\{0\}$ and $N \neq$ $H^{2}$, and let $\varphi(z) \in H^{\infty}\left(\Gamma_{z}\right)$ be a nonconstant function. We write operators $T_{\varphi}$ and $T_{w}^{*}$ on $H^{2}=M \oplus N$ in the matrix forms as

$$
T_{\varphi}=\left(\begin{array}{cc}
* & \left.P_{M} T_{\varphi}\right|_{N} \\
0 & S_{\varphi}
\end{array}\right), T_{w}^{*}=\left(\begin{array}{cc}
* & 0 \\
\left.P_{N} T_{w}^{*}\right|_{M} & S_{w}^{*}
\end{array}\right) \quad \text { on } H^{2}=\left(\begin{array}{c}
M \\
\oplus \\
N
\end{array}\right) .
$$

Let

$$
A=\left.P_{M} T_{\varphi}\right|_{N} \quad \text { and } \quad B=\left.P_{N} T_{w}^{*}\right|_{M} .
$$

Since $T_{\varphi} T_{w}^{*}=T_{w}^{*} T_{\varphi}$ on $H^{2}$, we have

$$
S_{\varphi} S_{w}^{*}=B A+S_{w}^{*} S_{\varphi}
$$

Hence we get the following.

Lemma 2.1. $\left[S_{\varphi}, S_{w}^{*}\right]=0$ if and only if $B A=0$.

It is not difficult to see that

$$
\begin{aligned}
\operatorname{ker} B & =\left\{f \in M: T_{w}^{*} f \in M\right\} \\
& =\left\{f \in M \ominus w M: T_{w}^{*} f=0\right\} \oplus w M \\
& =\left(M \cap H^{2}\left(\Gamma_{z}\right)\right) \oplus w M
\end{aligned}
$$

and

$$
\overline{\text { range } A}=M \ominus \operatorname{ker} A^{*}=M \ominus\left\{f \in M: T_{\varphi}^{*} f \in M\right\} .
$$

Then by Lemma 2.1, we have the following.

Lemma 2.2. $\left[S_{\varphi}, S_{w}^{*}\right]=0$ if and only if

$$
M \ominus\left\{f \in M: T_{\varphi}^{*} f \in M\right\} \subset\left(M \cap H^{2}\left(\Gamma_{z}\right)\right) \oplus w M .
$$

Lemma 2.3. If $\left[S_{\varphi}, S_{w}^{*}\right]=0$ and $\left[S_{z}, S_{w}^{*}\right] \neq 0$, then $M \cap H^{2}\left(\Gamma_{z}\right)$ is a nontrivial invariant subspace of $H^{2}\left(\Gamma_{z}\right)$. 
Proof. Since $M \neq H^{2}$, trivially $M \cap H^{2}\left(\Gamma_{z}\right) \neq H^{2}\left(\Gamma_{z}\right)$ holds. Suppose that $M \cap H^{2}\left(\Gamma_{z}\right)=\{0\}$. By Lemma 2.2,

$$
M \ominus\left\{f \in M: T_{\varphi}^{*} f \in M\right\} \subset w M .
$$

Hence

$$
M \ominus w M \subset\left\{f \in M: T_{\varphi}^{*} f \in M\right\} .
$$

Since $T_{w} T_{\varphi}^{*}=T_{\varphi}^{*} T_{w}$ on $H^{2}$, if $f \in M$ and $T_{\varphi}^{*} f \in M$, then $T_{\varphi}^{*}\left(w^{n} f\right)=w^{n} T_{\varphi}^{*} f \in$ $M$ for every $n \geq 0$, so that by the above we get

$$
w^{n}(M \ominus w M) \subset\left\{f \in M: T_{\varphi}^{*} f \in M\right\} .
$$

Therefore

$$
M=\sum_{n=0}^{\infty} \oplus w^{n}(M \ominus w M) \subset\left\{f \in M: T_{\varphi}^{*} f \in M\right\} .
$$

Thus we get $T_{\varphi}^{*} M \subset M$. This shows that $\varphi(z) N \subset N$.

Let

$$
\mathcal{A}=\left\{\psi(z) \in H^{\infty}\left(\Gamma_{z}\right): \psi N \subset N\right\} .
$$

Then both functions 1 and $\varphi(z)$ are contained in $\mathcal{A}$. For $\psi \in \mathcal{A}$ and $h \in N$, we have

$$
N \ni T_{z}^{*}(\psi h)=\left(T_{z}^{*} \psi\right) h+\psi(0) T_{z}^{*} h .
$$

Hence $\left(T_{z}^{*} \psi\right) N \subset N$, so that $T_{z}^{*} \mathcal{A} \subset \mathcal{A}$. It is easy to see that $\mathcal{A}$ is a weak-* closed subalgebra of $H^{\infty}\left(\Gamma_{z}\right)$. Let

$$
L=\left\{f(z) \in H^{1}\left(\Gamma_{z}\right): \int_{0}^{2 \pi} f\left(e^{i \theta} \overline{\psi\left(e^{i \theta}\right)} \frac{d \theta}{2 \pi}=0 \text { for every } \psi(z) \in \mathcal{A}\right\} .\right.
$$

Then $L$ is a closed subspace of $H^{1}\left(\Gamma_{z}\right)$. Since $T_{z}^{*} \mathcal{A} \subset \mathcal{A}$ and $1 \in \mathcal{A}$, we have $z L \subset L$.

Suppose that $L \neq\{0\}$. By the Beurling theorem, $L=q(z) H^{1}\left(\Gamma_{z}\right)$ for an inner function $q(z)$. Since $1 \in \mathcal{A}, q(0)=0$. Hence $\bar{z} q(z) \in H^{\infty}\left(\Gamma_{z}\right)$. Since $\varphi(z)^{n} \in \mathcal{A}$ for $n \geq 1$,

$$
\int_{0}^{2 \pi} e^{-i \theta} q\left(e^{i \theta}\right) \overline{\varphi\left(e^{i \theta}\right)} n e^{i \theta} h\left(e^{i \theta}\right) \frac{d \theta}{2 \pi}=\int_{0}^{2 \pi} q\left(e^{i \theta}\right) h\left(e^{i \theta}\right) \overline{\varphi\left(e^{i \theta}\right)} n \frac{d \theta}{2 \pi}=0
$$

for every $h(z) \in H^{1}\left(\Gamma_{z}\right)$. Hence $\bar{z} q(z) \overline{\varphi(z)}^{n} \in H^{\infty}\left(\Gamma_{z}\right)$ for every $n \geq 1$. By the Schneider theorem [11], we have $\overline{\varphi(z)} \in H^{\infty}\left(\Gamma_{z}\right)$. This shows that $\varphi(z)$ is constant. Since we assumed that $\varphi(z)$ is nonconstant, this is a contradiction. Therefore $L=\{0\}$. Hence $\mathcal{A}=H^{\infty}\left(\Gamma_{z}\right)$. Especially, we have $z \in \mathcal{A}$ and $z N \subset N$. Then $\left.T_{z}\right|_{N}=S_{z}$. Since $\left.T_{w}^{*}\right|_{N}=S_{w}^{*}$ and $T_{z} T_{w}^{*}=T_{w}^{*} T_{z}$ on $H^{2}$, we have $S_{z} S_{w}^{*}=S_{w}^{*} S_{z}$. This is a desired contradiction.

In the rest of this section, we assume that $M \cap H^{2}\left(\Gamma_{z}\right) \neq\{0\}$. Since $M \neq H^{2}$, $M \cap H^{2}\left(\Gamma_{z}\right) \neq H^{2}\left(\Gamma_{z}\right)$. By the Beurling theorem,

$$
M \cap H^{2}\left(\Gamma_{z}\right)=\theta(z) H^{2}\left(\Gamma_{z}\right)
$$


for some nonconstant inner function $\theta(z)$. Hence $\theta(z) H^{2} \subset M$. Write

$$
M_{\theta}=M \ominus \theta(z) H^{2} \text {. }
$$

Then

$$
M=M_{\theta} \oplus \theta(z) H^{2} \quad \text { and } \quad H^{2} \ominus \theta(z) H^{2}=M_{\theta} \oplus N .
$$

By the definition of $M_{\theta}$, we have $w M_{\theta} \subset M_{\theta}$ and $M_{\theta} \cap H^{2}\left(\Gamma_{z}\right)=\{0\}$. Note that if $\left[S_{\varphi}, S_{w}^{*}\right]=0$ and $\left[S_{z}, S_{w}^{*}\right] \neq 0$, then $M_{\theta} \neq\{0\}$. For, if $M_{\theta}=\{0\}$, then $M=\theta(z) H^{2}$ and $N=H^{2} \ominus \theta(z) H^{2}$. Then we have $\left[S_{z}, S_{w}^{*}\right]=0$, see [6], and this is a contradiction.

Lemma 2.4. Let $f \in M_{\theta}$. Then $T_{w}^{*} f \in M_{\theta}$ if and only if $f \in w M_{\theta}$.

Proof. Suppose that $T_{w}^{*} f \in M_{\theta}$. Then

$$
f-f(z, 0) \in w M_{\theta} \subset M_{\theta} .
$$

Since $f \in M_{\theta}, f(z, 0) \in M_{\theta}$. Since $M_{\theta} \cap H^{2}\left(\Gamma_{z}\right)=\{0\}, f(z, 0)=0$. Hence $f \in w M_{\theta}$. The converse is trivial.

Let $P_{\theta}$ be the orthogonal projection from $H^{2}$ onto $H^{2} \ominus \theta(z) H^{2}$, and $Q_{\varphi}$ be the operator on $H^{2} \ominus \theta(z) H^{2}$ defined by $Q_{\varphi} f=P_{\theta}(\varphi f)$ for $f \in H^{2} \ominus \theta(z) H^{2}$. We can write both operators $Q_{\varphi}$ and $\left.T_{w}^{*}\right|_{\left(H^{2} \ominus \theta H^{2}\right)}$ as

$$
Q_{\varphi}=\left(\begin{array}{cc}
* & \left.P_{M_{\theta}} T_{\varphi}\right|_{N} \\
0 & S_{\varphi}
\end{array}\right) \quad \text { on } \quad H^{2} \ominus \theta(z) H^{2}=\left(\begin{array}{c}
M_{\theta} \\
\oplus \\
N
\end{array}\right)
$$

and

$$
\left.T_{w}^{*}\right|_{\left(H^{2} \ominus \theta H^{2}\right)}=\left(\begin{array}{cc}
* & 0 \\
\left.P_{N} T_{w}^{*}\right|_{M_{\theta}} & S_{w}^{*}
\end{array}\right) \quad \text { on } \quad H^{2} \ominus \theta(z) H^{2}=\left(\begin{array}{c}
M_{\theta} \\
\oplus \\
N
\end{array}\right) .
$$

Let

$$
A_{\theta}=\left.P_{M_{\theta}} T_{\varphi}\right|_{N} \quad \text { and } \quad B_{\theta}=\left.P_{N} T_{w}^{*}\right|_{M_{\theta}} .
$$

Lemma 2.5. $\left[S_{\varphi}, S_{w}^{*}\right]=0$ if and only if $B_{\theta} A_{\theta}=0$.

Proof. Let $f \in H^{2} \ominus \theta(z) H^{2}=M_{\theta} \oplus N$. We have $T_{w}^{*}(\varphi(z) f)=\varphi(z) T_{w}^{*} f$. Write

$$
\varphi(z) f=Q_{\varphi} f \oplus f_{1} \in\left(M_{\theta} \oplus N\right) \oplus \theta(z) H^{2} .
$$

Since $T_{w}^{*} f_{1} \in \theta(z) H^{2}$ and $T_{w}^{*}\left(Q_{\varphi} f\right) \perp \theta(z) H^{2}$, we get $T_{w}^{*} Q_{\varphi} f=Q_{\varphi} T_{w}^{*} f$. Thus $Q_{\varphi} T_{w}^{*}=T_{w}^{*} Q_{\varphi}$ on $M_{\theta} \oplus N$. Similarly as Lemma 2.1, we can prove the assertion.

The following is a slight generalization of [7, Theorem 4.4].

Theorem 2.6. The following conditions are equivalent;

(i) $\left[S_{\varphi}, S_{w}^{*}\right]=0$,

(ii) $M_{\theta} \ominus\left\{f \in M_{\theta}: T_{\varphi}^{*} f \in M_{\theta}\right\} \subset w M_{\theta}$,

(iii) $T_{\varphi}^{*} M_{\theta} \subset M_{\theta}$, 
(iv) $\varphi(z) N \subset N \oplus \theta(z) H^{2}$.

Proof. By Lemma 2.4,

$$
\operatorname{ker} B_{\theta}=\left\{f \in M_{\theta}: T_{w}^{*} f \in M_{\theta}\right\}=w M_{\theta} .
$$

Also we have

$$
\overline{\text { range } A_{\theta}}=M_{\theta} \ominus \operatorname{ker} A_{\theta}^{*}=M_{\theta} \ominus\left\{f \in M_{\theta}: T_{\varphi}^{*} f \in M_{\theta}\right\} .
$$

Hence by Lemma 2.5, we get (i) $\Leftrightarrow$ (ii).

If (ii) holds, then

$$
M_{\theta} \ominus w M_{\theta} \subset\left\{f \in M_{\theta}: T_{\varphi}^{*} f \in M_{\theta}\right\} .
$$

Hence for each $n \geq 0$, we have

$$
T_{\varphi(z)}^{*} w^{n}\left(M_{\theta} \ominus w M_{\theta}\right)=w^{n} T_{\varphi(z)}^{*}\left(M_{\theta} \ominus w M_{\theta}\right) \subset w^{n} M_{\theta} \subset M_{\theta} .
$$

Since

$$
M_{\theta}=\sum_{n=0}^{\infty} \oplus w^{n}\left(M_{\theta} \ominus w M_{\theta}\right),
$$

we have $T_{\varphi}^{*} M_{\theta} \subset M_{\theta}$. Thus we get (iii).

(iii) $\Rightarrow$ (ii) is trivial.

It is not difficult to see that (iii) $\Leftrightarrow$ (iv).

Suppose that $\left[S_{\varphi}, S_{w}^{*}\right]=0$ and $\left[S_{z}, S_{w}^{*}\right] \neq 0$. Then we proved that

$$
\theta(z) H^{2} \varsubsetneqq M \quad \text { and } \quad \varphi(z)\left(H^{2} \ominus M\right) \subset\left(H^{2} \ominus M\right) \oplus \theta(z) H^{2} .
$$

Note that $\theta(z) H^{2}$ and $M$ are invariant subspaces of $H^{2}$. Now we fix an inner function $\theta(z)$. Here we have a question for which $\varphi(z) \in H^{\infty}\left(\Gamma_{z}\right)$ satisfies the above condition. In the next section, we study a similar question in the one variable Hardy space $H^{2}\left(\Gamma_{z}\right)$. In Section 4, we revisit on this question.

\section{A theorem on the unit circle}

In this section, we prove the following theorem.

Theorem 3.1. Let $N_{1}, N_{2}$ be backward shift invariant subspaces of $H^{2}\left(\Gamma_{z}\right)$ with $0 \neq N_{2} \varsubsetneqq N_{1} \neq H^{2}\left(\Gamma_{z}\right)$, and $\varphi(z) \in N_{1}$. Then

$$
\varphi\left(N_{2} \cap H^{\infty}\left(\Gamma_{z}\right)\right) \subset N_{2} \oplus\left(H^{2}\left(\Gamma_{z}\right) \ominus N_{1}\right)
$$

if and only if $\varphi(z)=c P_{N_{1}} 1$ for some $c \in \mathbb{C}$. In this case, if we define the operator $S_{\varphi}$ on $N_{1}$ by $S_{\varphi} f=P_{N_{1}}(\varphi f)$ for $f \in N_{1}$, then $S_{\varphi}=c I$.

To prove the theorem, we need two lemmas which are not difficult to show.

Lemma 3.2. Let $N$ be a backward shift invariant subspace of $H^{2}\left(\Gamma_{z}\right)$. Then $N \cap H^{\infty}\left(\Gamma_{z}\right)$ is dense in $N$.

Lemma 3.3. Let $N$ be a backward shift invariant subspace of $H^{2}\left(\Gamma_{z}\right)$ with $N \neq\{0\}$ and $N \neq H^{2}\left(\Gamma_{z}\right)$. If $\varphi \in H^{2}\left(\Gamma_{z}\right)$ is a nonconstant function, then $\varphi\left(N \cap H^{\infty}\left(\Gamma_{z}\right)\right) \not \subset N$. 
Proof of Theorem 3.1. By the Beurling theorem,

$$
H^{2}\left(\Gamma_{z}\right) \ominus N_{1}=\theta H^{2}\left(\Gamma_{z}\right)
$$

for some nonconstant inner function $\theta$.

First, suppose that

$$
\varphi\left(N_{2} \cap H^{\infty}\left(\Gamma_{z}\right)\right) \subset N_{2} \oplus\left(H^{2}\left(\Gamma_{z}\right) \ominus N_{1}\right) .
$$

Since $N_{2} \neq\{0\}$, by Lemma 3.2 there exists $h_{1} \in N_{2} \cap H^{\infty}\left(\Gamma_{z}\right)$ with $h_{1}(0)=1$. Write

$$
\varphi h_{1}=f_{1} \oplus \theta g_{1} \in N_{2} \oplus\left(H^{2}\left(\Gamma_{z}\right) \ominus N_{1}\right)=N_{2} \oplus \theta H^{2}\left(\Gamma_{z}\right) .
$$

Also for each $h \in N_{2} \cap H^{\infty}\left(\Gamma_{z}\right)$, we can write

$$
\varphi h=f \oplus \theta g \in N_{2} \oplus \theta H^{2}\left(\Gamma_{z}\right) .
$$

When $h(0)=0$, we shall prove that

$$
g(0)=0 .
$$

Since

by (3.2) we have

$$
T_{z}^{*}(\varphi h)=\varphi T_{z}^{*} h+h(0) T_{z}^{*} \varphi=\varphi T_{z}^{*} h,
$$

$$
\begin{aligned}
\varphi T_{z}^{*} h & =T_{z}^{*}(\varphi h)=T_{z}^{*}(f+\theta g) \\
& =T_{z}^{*} f+\theta T_{z}^{*} g+g(0) T_{z}^{*} \theta \\
& =\left(T_{z}^{*} f+g(0) T_{z}^{*} \theta\right)+\theta T_{z}^{*} g .
\end{aligned}
$$

Note that $T_{z}^{*} h \in N_{2} \cap H^{\infty}\left(\Gamma_{z}\right)$ and $T_{z}^{*} f+g(0) T_{z}^{*} \theta \perp \theta H^{2}\left(\Gamma_{z}\right)$. By the assumption, $\varphi T_{z}^{*} h \in N_{2} \oplus \theta H^{2}\left(\Gamma_{z}\right)$. Hence

$$
T_{z}^{*} f+g(0) T_{z}^{*} \theta \in N_{2} \text {. }
$$

Since $T_{z}^{*} f \in N_{2}, g(0) T_{z}^{*} \theta \in N_{2}$. To prove (3.3), suppose that $g(0) \neq 0$. Then $T_{z}^{*} \theta \in N_{2}$. Let $N$ be a backward shift invariant subspace generated by $T_{z}^{*} \theta$. Since $N_{1}=H^{2}\left(\Gamma_{z}\right) \ominus \theta H^{2}\left(\Gamma_{z}\right)$, we have $N=N_{1}$. Since $T_{z}^{*} \theta \in N_{2}, N \subset N_{2}$. This contradicts $N_{2} \varsubsetneqq N_{1}$. Therefore $g(0)=0$. Thus we get (3.3).

By (3.1) and (3.2),

$$
\varphi\left(h-h(0) h_{1}\right)=\left(f-h(0) f_{1}\right) \oplus \theta\left(g-h(0) g_{1}\right) \in N_{2} \oplus \theta H^{2}\left(\Gamma_{z}\right) .
$$

Since $\left(h-h(0) h_{1}\right)(0)=0$, by $(3.3)$ we get

$$
g(0)=h(0) g_{1}(0) \text {. }
$$

By (3.2) again,

$$
\varphi T_{z}^{*} h+h(0) T_{z}^{*} \varphi=T_{z}^{*}(\varphi h)=\left(T_{z}^{*} f+g(0) T_{z}^{*} \theta\right)+\theta T_{z}^{*} g,
$$

so that

$$
\varphi T_{z}^{*} h=\left(-h(0) T_{z}^{*} \varphi+T_{z}^{*} f+g(0) T_{z}^{*} \theta\right) \oplus \theta T_{z}^{*} g .
$$

Since $T_{z}^{*} h \in N_{2} \cap H^{\infty}\left(\Gamma_{z}\right)$ and $\varphi \perp \theta H^{2}\left(\Gamma_{z}\right)$, by the assumption we have

$$
-h(0) T_{z}^{*} \varphi+T_{z}^{*} f+g(0) T_{z}^{*} \theta \in N_{2} .
$$


Similarly we have

$$
\begin{gathered}
\varphi T_{z}^{* 2} h=\left(-\left(T_{z}^{*} h\right)(0) T_{z}^{*} \varphi-h(0) T_{z}^{* 2} \varphi+T_{z}^{* 2} f+g(0) T_{z}^{* 2} \theta\right. \\
\left.+\left(T_{z}^{*} g\right)(0) T_{z}^{*} \theta\right) \oplus \theta T_{z}^{* 2} g
\end{gathered}
$$

Repeating the same argument, we get

$$
\begin{array}{r}
\varphi T_{z}^{* n} h=\left[-\left(\sum_{j=0}^{n-1}\left(T_{z}^{*(n-j-1)} h\right)(0) T_{z}^{*(j+1)} \varphi\right)+T_{z}^{* n} f\right. \\
\left.+\left(\sum_{j=0}^{n-1}\left(T_{z}^{* j} g\right)(0) T_{z}^{*(n-j)} \theta\right)\right] \oplus \theta T_{z}^{* n} g .
\end{array}
$$

Since $h \in N_{2} \cap H^{\infty}\left(\Gamma_{z}\right), T_{z}^{* n} h \in N_{2} \cap H^{\infty}\left(\Gamma_{z}\right)$. Hence by (3.2) and (3.4),

$$
\left(T_{z}^{* n} g\right)(0)=\left(T_{z}^{* n} h\right)(0) g_{1}(0)
$$

for every $n \geq 0$. This shows that $g=g_{1}(0) h$. By (3.2), we obtain

$$
\left(\varphi-g_{1}(0) \theta\right) h=f \in N_{2}
$$

for every $h \in N_{2} \cap H^{\infty}\left(\Gamma_{z}\right)$. By Lemma 3.3, $\varphi-g_{1}(0) \theta$ is constant. Write $\varphi-g_{1}(0) \theta=c$. Since $\varphi \in N_{1}$, we have $\varphi=c P_{N_{1}} 1$.

Next, suppose that $\varphi=c P_{N_{1}} 1$. Then

$$
\varphi=c P_{N_{1}} 1=c(1-\overline{\theta(0)} \theta) .
$$

Hence for $f \in N_{2} \cap H^{\infty}\left(\Gamma_{z}\right)$, we have

$$
\varphi f=c f-c \overline{\theta(0)} \theta f \in N_{2} \oplus \theta H^{2}\left(\Gamma_{z}\right) .
$$

Thus we get $\varphi\left(N_{2} \cap H^{\infty}\left(\Gamma_{z}\right)\right) \subset N_{2} \oplus\left(H^{2}\left(\Gamma_{z}\right) \ominus N_{1}\right)$.

Corollary 3.4. Let $N_{1}, N_{2}$ be backward shift invariant subspaces of $H^{2}\left(\Gamma_{z}\right)$ with $\{0\} \neq N_{2} \varsubsetneqq N_{1} \neq H^{2}\left(\Gamma_{z}\right)$, and $\varphi(z) \in L^{\infty}\left(\Gamma_{z}\right)$. Define the operator $S_{\varphi}$ on $N_{1}$ by $S_{\varphi} h=P_{N_{1}}(\varphi h)$ for $h \in N_{1}$. Then $S_{\varphi} N_{2} \subset N_{2}$ if and only if

$$
\varphi \in \mathbb{C}+H^{2}\left(\Gamma_{z}\right)^{\perp}+\left(H^{2}\left(\Gamma_{z}\right) \ominus N_{1}\right)=\overline{H^{2}\left(\Gamma_{z}\right)}+\left(H^{2}\left(\Gamma_{z}\right) \ominus N_{1}\right) .
$$

Proof. Write $H^{2}\left(\Gamma_{z}\right) \ominus N_{1}=\theta H^{2}\left(\Gamma_{z}\right)$ for some inner function $\theta$. Let

$$
\varphi=\varphi_{1} \oplus \varphi_{2} \oplus \theta \varphi_{3} \in H^{2}\left(\Gamma_{z}\right)^{\perp} \oplus N_{1} \oplus \theta H^{2}\left(\Gamma_{z}\right) .
$$

It is easy to see that

$$
P_{N_{1}}\left(\varphi_{1}\left(N_{2} \cap H^{\infty}\left(\Gamma_{z}\right)\right)\right) \subset N_{2}
$$

and

$$
P_{N_{1}}\left(\theta \varphi_{3}\left(N_{2} \cap H^{\infty}\left(\Gamma_{z}\right)\right)\right)=\{0\} .
$$


Hence $S_{\varphi} N_{2} \subset N_{2}$ if and only if $P_{N_{1}}\left(\varphi_{2}\left(N_{2} \cap H^{\infty}\left(\Gamma_{z}\right)\right)\right) \subset N_{2}$. By Theorem $3.1, S_{\varphi} N_{2} \subset N_{2}$ if and only if

$$
\begin{aligned}
\varphi & =\varphi_{1}+c P_{N_{1}} 1+\theta \varphi_{3} \\
& =\varphi_{1}+c(1-\overline{\theta(0)} \theta)+\theta \varphi_{3} \\
& =\varphi_{1}+c+\theta\left(\varphi_{3}-c \overline{\theta(0)}\right) .
\end{aligned}
$$

This completes the proof.

The following corollaries follow from Corollary 3.4 directly.

Corollary 3.5. Let $N_{1}, N_{2}$ be backward shift invariant subspaces of $H^{2}\left(\Gamma_{z}\right)$ with $\{0\} \neq N_{2} \varsubsetneqq N_{1} \neq H^{2}\left(\Gamma_{z}\right)$, and $\varphi(z) \in H^{\infty}\left(\Gamma_{z}\right)$. Then $\varphi N_{2} \subset N_{2} \oplus$ $\left(H^{2}\left(\Gamma_{z}\right) \ominus N_{1}\right)$ if and only if $\varphi \in \mathbb{C}+\left(H^{2}\left(\Gamma_{z}\right) \ominus N_{1}\right)$.

Corollary 3.6. Let $N_{1}, N_{2}$ be backward shift invariant subspaces of $H^{2}\left(\Gamma_{z}\right)$ with $\{0\} \neq N_{2} \subset N_{1} \neq H^{2}\left(\Gamma_{z}\right)$, and $\varphi(z) \in H^{\infty}\left(\Gamma_{z}\right)$. If $\varphi N_{2} \subset N_{2} \oplus\left(H^{2}\left(\Gamma_{z}\right) \ominus\right.$ $\left.N_{1}\right)$, then $N_{1}=N_{2}$ if and only if $\varphi \notin \mathbb{C}+\left(H^{2}\left(\Gamma_{z}\right) \ominus N_{1}\right)$.

Corollary 3.7. Let $M_{1}, M_{2}$ be invariant subspaces of $H^{2}\left(\Gamma_{z}\right)$ with $\{0\} \neq M_{1} \varsubsetneqq$ $M_{2} \neq H^{2}\left(\Gamma_{z}\right)$, and $\varphi(z) \in H^{\infty}\left(\Gamma_{z}\right)$. Then $T_{\varphi}^{*}\left(M_{2} \ominus M_{1}\right) \subset M_{2} \ominus M_{1}$ if and only if $\varphi \in \mathbb{C}+M_{1}$.

Corollary 3.8. Let $M_{1}, M_{2}$ be invariant subspaces of $H^{2}\left(\Gamma_{z}\right)$ with $\{0\} \neq M_{1} \varsubsetneqq$ $M_{2} \subset H^{2}\left(\Gamma_{z}\right)$, and $\varphi(z) \in H^{\infty}\left(\Gamma_{z}\right)$. If $T_{\varphi}^{*}\left(M_{2} \ominus M_{1}\right) \subset M_{2} \ominus M_{1}$, then $\varphi \notin \mathbb{C}+M_{1}$ if and only if $M_{2}=H^{2}\left(\Gamma_{z}\right)$.

\section{The main theorem}

As applications of the results in Sections 2 and 3, we prove the following.

Theorem 4.1. Let $N$ be a backward shift invariant subspace of $H^{2}$ with $N \neq$ $\{0\}$ and $N \neq H^{2}$. Let $\varphi(z) \in H^{\infty}\left(\Gamma_{z}\right)$ be a nonconstant function. If $\left[S_{\varphi}, S_{w}^{*}\right]=$ 0 and $\left[S_{z}, S_{w}^{*}\right] \neq 0$, then $\varphi(z)-c \in M \cap H^{\infty}\left(\Gamma_{z}\right)$ for some $c \in \mathbb{C}$ and $S_{\varphi}=c I$.

Proof. By Lemma 2.3, $M \cap H^{2}\left(\Gamma_{z}\right)=\theta(z) H^{2}\left(\Gamma_{z}\right)$ for a nonconstant inner function $\theta(z)$. Since $\theta(z) H^{2} \subset M$, as in Section 2 we write

$$
M_{\theta}=M \ominus \theta(z) H^{2} .
$$

Since $\left[S_{z}, S_{w}^{*}\right] \neq 0$, we have $M_{\theta} \neq\{0\}$. By Theorem 2.6,

$$
\varphi(z) N \subset N \oplus \theta(z) H^{2}
$$

and

$$
T_{\varphi}^{*} M_{\theta} \subset M_{\theta} .
$$

To prove the assertion, we assume that

$$
\varphi(z)-c \notin \theta(z) H^{\infty}\left(\Gamma_{z}\right)
$$

for every $c \in \mathbb{C}$. We shall prove that $\left[S_{z}, S_{w}^{*}\right]=0$. This will be a desired contradiction. We consider two cases $\theta(0)=0$ and $\theta(0) \neq 0$ separately. 
Case 1. Suppose that $\theta(0)=0$. If $\theta(z)=c z$ for some constant $c$ with $|c|=1$, then it is easy to see that

$$
M=\theta(z) H^{2}+q(w) H^{2}
$$

for either a nonconstant inner function $q(w)$ or $q(w)=0$. In this case, by [6] we have $\left[S_{z}, S_{w}^{*}\right]=0$. So, we may assume that $\theta(z)=z \theta_{1}(z)$ for a nonconstant inner function $\theta_{1}(z)$. Then

$$
H^{2} \ominus \theta(z) H^{2}=H^{2}\left(\Gamma_{w}\right) \oplus z\left(H^{2} \ominus \theta_{1}(z) H^{2}\right) .
$$

We divide the proof into two subcases.

Subcase 1.1. Assume that $\theta_{1}(z) M_{\theta} \subset \theta(z) H^{2}$. Then $M_{\theta} \subset z H^{2}$. Hence $H^{2}\left(\Gamma_{w}\right) \subset N$. For each nonnegative integer $n$, let

$$
L_{n}=\left\{f(z) \in H^{2}\left(\Gamma_{z}\right) \ominus \theta(z) H^{2}\left(\Gamma_{z}\right): w^{n} f(z) \in N\right\} .
$$

Then $1 \in L_{n}, L_{n}$ is a nonzero closed subspace of $H^{2}\left(\Gamma_{z}\right) \ominus \theta(z) H^{2}\left(\Gamma_{z}\right)$, and $T_{z}^{*} L_{n} \subset L_{n}$. By (4.2),

$$
w^{n} \varphi(z) L_{n} \subset \varphi(z) N \subset N \oplus \theta(z) H^{2},
$$

so we have

$$
\varphi(z) L_{n} \subset L_{n} \oplus \theta(z) H^{2}\left(\Gamma_{z}\right) .
$$

By (4.4) and Corollary 3.6, $L_{n}=H^{2}\left(\Gamma_{z}\right) \ominus \theta(z) H^{2}\left(\Gamma_{z}\right)$. Hence

$$
w^{n}\left(H^{2}\left(\Gamma_{z}\right) \ominus \theta(z) H^{2}\left(\Gamma_{z}\right)\right) \subset N
$$

for every $n \geq 0$. Therefore

$$
H^{2} \ominus \theta(z) H^{2}=\sum_{n=0}^{\infty} \oplus w^{n}\left(H^{2}\left(\Gamma_{z}\right) \ominus \theta(z) H^{2}\left(\Gamma_{z}\right)\right) \subset N .
$$

By (4.1), $H^{2} \ominus \theta(z) H^{2}=M_{\theta} \oplus N$, so that $M_{\theta}=\{0\}$. This contradicts $\left[S_{z}, S_{w}^{*}\right] \neq$ 0 .

Subcase 1.2. Assume that $\theta_{1}(z) M_{\theta} \not \subset \theta(z) H^{2}$. By (4.5), for every $g \in M_{\theta}$ we can write

$$
g=f_{g}(w) \oplus z h_{g}(z, w),
$$

where $f_{g} \in H^{2}\left(\Gamma_{w}\right)$ and $h_{g} \in H^{2} \ominus \theta_{1}(z) H^{2}$. Since $\theta_{1}(z) M_{\theta} \subset M$, we have

$$
\theta_{1}(z) g=\theta_{1}(z) f_{g}(w) \oplus z \theta_{1}(z) h_{g}(z, w) \in M=M_{\theta} \oplus \theta(z) H^{2},
$$

so that $\theta_{1}(z) f_{g}(w) \in M_{\theta}$. Since $\theta_{1}(z) M_{\theta} \not \subset \theta(z) H^{2}, f_{g}(w) \neq 0$ for some $g \in M_{\theta}$. Then $\left\{f_{g}(w): g \in M_{\theta}\right\} \neq\{0\}$. Since $w M_{\theta} \subset M_{\theta}$, by (4.6) $\overline{\left\{f_{g}(w): g \in M_{\theta}\right\}}$ is a nonzero $T_{w}$-invariant subspace of $H^{2}\left(\Gamma_{w}\right)$. Hence there is a one variable inner function $q(w)$ such that

$$
q(w) H^{2}\left(\Gamma_{w}\right)=\overline{\left\{f_{g}(w): g \in M_{\theta}\right\}} .
$$


Since $\theta_{1}(z)\left\{f_{g}(w): g \in M_{\theta}\right\} \subset M_{\theta}$, we have

$$
\theta_{1}(z) q(w) H^{2}\left(\Gamma_{w}\right) \subset M_{\theta} .
$$

If $q(w)$ is constant, then $\theta_{1}(z) \in M_{\theta}$ and

$$
\theta(z) H^{2}\left(\Gamma_{z}\right) \varsubsetneqq \mathbb{C} \cdot \theta_{1}(z)+\theta(z) H^{2}\left(\Gamma_{z}\right) \subset M \cap H^{2}\left(\Gamma_{z}\right),
$$

so that $\theta(z) H^{2}\left(\Gamma_{z}\right) \neq M \cap H^{2}\left(\Gamma_{z}\right)$. This is a contradiction. Hence $q(w)$ is nonconstant. By (4.6) and (4.7), we get

$$
\left(H^{2}\left(\Gamma_{w}\right) \ominus q(w) H^{2}\left(\Gamma_{w}\right)\right) \perp M_{\theta} .
$$

For each nonnegative integer $n$, let

$$
L_{n}=\left\{f(z) \in H^{2}\left(\Gamma_{z}\right) \ominus \theta(z) H^{2}\left(\Gamma_{z}\right): f(z) w^{n} q(w) \in M_{\theta}\right\} .
$$

By (4.8), $\theta_{1}(z) \in L_{n}$. Since $z M_{\theta} \subset M_{\theta} \oplus \theta(z) H^{2}, L_{n} \oplus \theta(z) H^{2}\left(\Gamma_{z}\right)$ is an invariant subspace of $H^{2}\left(\Gamma_{z}\right)$. By (4.3), we have $T_{\varphi}^{*} L_{n} \subset L_{n}$. By (4.4) and Corollary 3.8, $L_{n}=H^{2}\left(\Gamma_{z}\right) \ominus \theta(z) H^{2}\left(\Gamma_{z}\right)$. Hence

$$
w^{n} q(w)\left(H^{2}\left(\Gamma_{z}\right) \ominus \theta(z) H^{2}\left(\Gamma_{z}\right)\right) \subset M_{\theta}
$$

for every $n \geq 0$. Thus we get

$$
q(w)\left(H^{2} \ominus \theta(z) H^{2}\right) \subset M_{\theta} .
$$
let

By $(4.9), H^{2}\left(\Gamma_{w}\right) \ominus q(w) H^{2}\left(\Gamma_{w}\right) \subset N$. For each $\psi(w) \in H^{2}\left(\Gamma_{w}\right) \ominus q(w) H^{2}\left(\Gamma_{w}\right)$,

$$
L_{\psi}=\left\{f(z) \in H^{2}\left(\Gamma_{z}\right) \ominus \theta(z) H^{2}\left(\Gamma_{z}\right): f(z) \psi(w) \in N\right\} .
$$

Then $1 \in L_{\psi}$, and in the same way as Subcase 1.1, $L_{\psi}$ is a nonzero closed subspace of $H^{2}\left(\Gamma_{z}\right) \ominus \theta(z) H^{2}\left(\Gamma_{z}\right)$ such that $T_{z}^{*} L_{\psi} \subset L_{\psi}$ and $\varphi(z) L_{\psi} \subset L_{\psi} \oplus$ $\theta(z) H^{2}\left(\Gamma_{z}\right)$. Hence by (4.4) and Corollary 3.6, $L_{\psi}=H^{2}\left(\Gamma_{z}\right) \ominus \theta(z) H^{2}\left(\Gamma_{z}\right)$. Therefore

$$
\psi(w)\left(H^{2}\left(\Gamma_{z}\right) \ominus \theta(z) H^{2}\left(\Gamma_{z}\right)\right) \subset N
$$

for every $\psi(w) \in H^{2}\left(\Gamma_{w}\right) \ominus q(w) H^{2}\left(\Gamma_{w}\right)$, and hence

$$
\left(H^{2} \ominus \theta(z) H^{2}\right) \ominus q(w)\left(H^{2} \ominus \theta(z) H^{2}\right) \subset N .
$$

Since $H^{2} \ominus \theta(z) H^{2}=M_{\theta} \oplus N$, by (4.10) and (4.11) we get

$$
N=\left(H^{2} \ominus \theta(z) H^{2}\right) \ominus q(w)\left(H^{2} \ominus \theta(z) H^{2}\right) .
$$

By [6], this shows that $\left[S_{z}, S_{w}^{*}\right]=0$.

Case 2. Suppose that $\theta(0) \neq 0$. Let $\varphi^{\prime}(z)=\varphi(z)-\langle\varphi, \theta\rangle \theta(z)$. Then $S_{\varphi}=S_{\varphi^{\prime}}$, so that we may assume that $\varphi \perp \theta$. Write

$$
\varphi(z)=\varphi_{1}(z)+\theta(z) z \varphi_{2}(z),
$$

where $\varphi_{1} \in H^{2}\left(\Gamma_{z}\right) \ominus \theta H^{2}\left(\Gamma_{z}\right)$ and $\varphi_{2} \in H^{2}\left(\Gamma_{z}\right)$. By $(4.4), \varphi_{1}(z) \neq 0$. Since $\theta(0) \neq 0, T_{z}^{*} \varphi_{1}(z) \neq 0$. For each $h \in N$, by (4.2) we can write

$$
\varphi h=f_{h}+\theta g_{h} \in N \oplus \theta H^{2} .
$$


Applying $T_{z}^{*}$ for the both side of the above, we have

$$
\varphi T_{z}^{*} h+h(0, w) T_{z}^{*} \varphi=T_{z}^{*} f_{h}+g(0, w) T_{z}^{*} \theta+\theta T_{z}^{*} g_{h} .
$$

Hence by (4.12),

$$
\begin{aligned}
\varphi T_{z}^{*} h & =-h(0, w) T_{z}^{*} \varphi+T_{z}^{*} f_{h}+g_{h}(0, w) T_{z}^{*} \theta+\theta T_{z}^{*} g_{h} \\
& =-h(0, w) T_{z}^{*} \varphi_{1}+T_{z}^{*} f_{h}+g_{h}(0, w) T_{z}^{*} \theta+\theta\left(T_{z}^{*} g_{h}-h(0, w) \varphi_{2}\right) .
\end{aligned}
$$

Note that

$$
-h(0, w) T_{z}^{*} \varphi_{1}+T_{z}^{*} f_{h}+g_{h}(0, w) T_{z}^{*} \theta \perp \theta H^{2} .
$$

Since $h \in N$, we have $T_{z}^{*} h \in N$, so that by (4.2) we have

$$
-h(0, w) T_{z}^{*} \varphi_{1}+T_{z}^{*} f_{h}+g_{h}(0, w) T_{z}^{*} \theta \in N .
$$

Since $f_{h} \in N$, also we have $T_{z}^{*} f_{h} \in N$ and

$$
-h(0, w) T_{z}^{*} \varphi_{1}+g_{h}(0, w) T_{z}^{*} \theta \in N .
$$

Write

$$
\Theta(z)=\theta^{2}(z)-\theta(0) \theta(z)
$$

We have

$$
T_{\theta \frac{\theta-\theta(0)}{z}}^{*} T_{z}^{*}=T_{\theta^{2}-\theta(0) \theta}^{*}=T_{\Theta}^{*} .
$$

Since

$$
\begin{gathered}
T_{\theta \frac{\theta-\theta(0)}{z}}^{*} N \subset N, \\
-h(0, w)\left(T_{\Theta}^{*} \varphi_{1}+a T_{\Theta}^{*} \theta\right)+g(0, w) T_{\Theta}^{*} \theta \in N .
\end{gathered}
$$

Since $\varphi_{1} \in N \subset H^{2} \ominus \theta H^{2}$, we have $T_{\Theta}^{*} \varphi_{1}=0$. Since $T_{\Theta}^{*} \theta=-\overline{\theta(0)}$, we get

$$
a \overline{\theta(0)} h(0, w)-\overline{\theta(0)} g(0, w) \in N .
$$

Since $\theta(0) \neq 0$,

$$
a h(0, w)-g(0, w) \in N
$$

Thus we get

$$
a h(0, w)-g(0, w) \perp \theta(z) H^{2} .
$$

Because $\theta(0) \neq 0$, we have $a h(0, w)-g(0, w)=0$. Hence by (4.9),

$$
-h(0, w) T_{z}^{*} \varphi_{1}(z) \in N \text {. }
$$

Note that $T_{z}^{*} \varphi_{1}(z) \neq 0$. In the same way as Subcase 1.2 ,

$$
h(0, w)\left(H^{2}\left(\Gamma_{z}\right) \ominus \theta(z) H^{2}\left(\Gamma_{z}\right)\right) \subset N \subset H^{2} \ominus \theta(z) H^{2}
$$

for every $h \in N$. Since $T_{w}^{*} N \subset N$ and $N \neq\{0\}, \overline{\{h(0, w): h \in N\}}$ is a nontrivial $T_{w}^{*}$-invariant subspace of $H^{2}\left(\Gamma_{w}\right)$, so that

$$
\overline{\{h(0, w): h \in N\}}=H^{2}\left(\Gamma_{w}\right) \ominus q(w) H^{2}\left(\Gamma_{w}\right)
$$

for either nontrivial inner function $q(w)$ or $q(w)=0$. Hence

$$
\left(H^{2} \ominus \theta(z) H^{2}\right) \ominus q(w)\left(H^{2} \ominus \theta(z) H^{2}\right) \subset N .
$$


For every $f \in N$, write

$$
f=\sum_{n=0}^{\infty} \oplus f_{n}(w) z^{n} .
$$

Since $T_{z}^{*} N \subset N, f_{n}(w) \in H^{2}\left(\Gamma_{w}\right) \ominus q(w) H^{2}\left(\Gamma_{w}\right)$ for every $n \geq 0$. Hence

$$
N \subset\left(H^{2} \ominus \theta(z) H^{2}\right) \ominus q(w)\left(H^{2} \ominus \theta(z) H^{2}\right) .
$$

Therefore

$$
N=\left(H^{2} \ominus \theta(z) H^{2}\right) \ominus q(w)\left(H^{2} \ominus \theta(z) H^{2}\right) .
$$

This shows that $\left[S_{z}, S_{w}^{*}\right]=0$. This completes the proof.

\section{References}

[1] H. Bercovici, Operator Theory and Arithmetic in $H^{\infty}$, Mathematical Surveys and Monographs, 26. American Mathematical Society, Providence, RI, 1988.

[2] A. Beurling, On two problems concerning linear transformations in Hilbert space, Acta Math. 81 (1948), 239-255.

[3] X. Chen and K. Guo, Analytic Hilbert Modules, Chapman \& Hall/CRC, Boca Raton, FL, 2003.

[4] K. J. Izuchi and K. H. Izuchi, Commutativity in two variable Jordan blocks on the Hardy space, preprint.

[5] Cross commutators on backward shift invariant subspaces over the bidisk, Acta Sci. Math. (Szeged) 72 (2006), no. 1-2, 251-270.

[6] K. J. Izuchi, T. Nakazi, and M. Seto, Backward shift invariant subspaces in the bidisc. II, J. Operator Theory 51 (2004), no. 2, 361-376.

[7] _ Backward shift invariant subspaces in the bidisc. III, Acta Sci. Math. (Szeged) 70 (2004), no. 3-4, 727-749.

[8] V. Mandrekar, The validity of Beurling theorems in polydiscs, Proc. Amer. Math. Soc. 103 (1988), no. 1, 145-148.

[9] N. Nikol'skii, Treatise on the Shift Operator, Springer-Verlag, Berlin, 1986.

[10] W. Rudin, Function Theory in Polydiscs, Benjamin, New York, 1969.

[11] R. Schneider, Isometries of $H^{p}\left(U^{n}\right)$, Canad. J. Math. 25 (1973), 92-95.

KEI JI IZUCHI

Department of Mathematics

NiIgATA University

NiIGATA 950-2181, JAPAN

E-mail address: izuchi@math.sc.niigata-u.ac.jp

Kou Hei Izuchi

FACULTY OF EDUCATION

YAMAGUCHI UNIVERSITY

YAMAGUCHI 753-8513, JAPAN

E-mail address: kh.izuchi@gmail.com 